\title{
MOTION IN THE NEIGHBORHOOD OF A STATIONARY POINT*
}

\author{
BY \\ J. A. MORRISON
}

Bell T'elephone Laboratories, Incorporated, Murray Hill, New Jersey

\begin{abstract}
A perturbed system of differential equations is considered, wherein the zero order rate of change of one variable, which depends on the other, slowly changing, variables, vanishes at a particular point. A detailed analysis of the motions in certain close neighborhoods of the stationary point of the perturbed system is given, the method of averaging being applied, after some preliminary transformations have been made. It is found that the motions corresponding to the averaged equations are damped under the stability conditions given in [1] by Volosov and Morgunov, who considered a more general problem. However, our analysis reveals the nature of the motion in the neighborhood of the stationary point, and, moreover, our results are valid for a time interval of the order of the reciprocal of the perturbation parameter, rather than the reciprocal of just its square root.
\end{abstract}

1. Introduction. In this paper we consider the perturbed system of equations

$$
\begin{aligned}
d x_{i} / d t & =X_{i}(\mathbf{x}, y ; \epsilon)=\epsilon X_{i}^{(1)}(\mathbf{x}, y)+\epsilon^{2} X_{i}^{(2)}(\mathbf{x}, y)+\cdots ; \\
d y / d t & =Y(\mathbf{x}, y ; \epsilon)=Y^{(0)}(\mathbf{x})+\epsilon Y^{(1)}(\mathbf{x}, y)+\epsilon^{2} Y^{(2)}(\mathbf{x}, y)+\cdots,
\end{aligned}
$$

where $\epsilon>0$ is a small parameter, and $i=1, \cdots, n$. It is supposed that

$$
X_{i}^{(1)}(0,0)=0 ; \quad Y^{(0)}(0)=0 .
$$

In a recent paper [1], Volosov and Morgunov start from a system of the form (1.1), wherein they take $y$ to be a vector also. However, the detailed results given in [1] are for the case when $y$ is scalar (the results for the more general case being very cumbersome), and we will confine our attention to this case.

Volosov and Morgunov suppose that, for sufficiently small $\epsilon$, the system (1.1) has a stationary point $\mathbf{x}=\alpha(\epsilon), y=\beta(\epsilon)$ where

and, letting

$$
\alpha_{i}(\epsilon)=\epsilon \alpha_{i}^{(1)}+\epsilon^{2} \alpha_{i}^{(2)}+\cdots, \quad \beta(\epsilon)=\epsilon \beta^{(1)}+\epsilon^{2} \beta^{(2)}+\cdots,
$$

$$
u_{i}=\left(x_{i}-\epsilon \alpha_{i}^{(1)}\right), \quad(i=1, \cdots, n) ; \quad u_{n+1}=y,
$$

they reduce the system (1.1) to one of the form

$$
d \mathfrak{u} / d t=A(\epsilon) \mathfrak{u}+\mathbf{F}(\mathfrak{u}, \boldsymbol{\epsilon}) .
$$

Here $A(\epsilon)$ is a matrix of order $n+1$, of the form

$$
A(\epsilon)=\left(\begin{array}{cccc}
\epsilon a_{11} & \cdots & \epsilon a_{1 n} & \epsilon b_{1} \\
\cdots & \cdots & \cdots & \cdots \\
\epsilon a_{n 1} & \cdots & \epsilon a_{n n} & \epsilon b_{n} \\
c_{1}+\epsilon d_{1} & \cdots & c_{n}+\epsilon d_{n} & \epsilon h
\end{array}\right),
$$

*Received March 6, 1967. 
where

$$
\begin{aligned}
a_{i j} & =\left(\partial X_{i}^{(1)} / \partial x_{j}\right)(0,0) ; & b_{i} & =\left(\partial X_{i}^{(1)} / \partial y\right)(0,0), \\
c_{i} & =\left(\partial Y^{(1)} / \partial x_{j}\right)(0) ; & h & =\left(\partial Y^{(1)} / \partial y\right)(0,0),
\end{aligned}
$$

and

$$
d_{i}=\alpha_{i}^{(1)} \frac{\partial^{2} Y^{(0)}}{\partial x_{i} \partial x_{i}}(0)+\frac{\partial Y^{(1)}}{\partial x_{i}}(0,0),
$$

the summation convention being understood. We have changed the notation somewhat, since we are considering only the case when $y$ is a scalar, and have corrected a misprint in the definition of $d_{i}$ (a factor $\frac{1}{2}$ appearing, in [1], in front of the term containing the second derivatives).

If the right hand sides of (1.1) are sufficiently smooth, then in (1.5)

$$
\begin{aligned}
F_{i} & =O\left(\epsilon^{2}+\epsilon^{2}\|\mathfrak{u}\|+\epsilon\|\mathbf{u}\|^{2}\right) \quad(i=1, \cdots, n) ; \\
F_{n+1} & =O\left(\epsilon^{2}+\epsilon^{2}\|\mathfrak{u}\|+\|\mathfrak{u}\|^{2}\right) .
\end{aligned}
$$

Instead of (1.5), Volosov and Morgunov considered a nonautonomous system of equations of the form

$$
d \mathrm{z} / d t=A(\epsilon) \mathbf{z}+\boldsymbol{\Phi}(\epsilon, \mathbf{z}, t)
$$

where

$$
\begin{aligned}
\Phi_{i} & =O\left(\epsilon^{2}+\epsilon^{2}\|z\|+\epsilon\|z\|^{2}\right) \quad(i=1, \cdots, n) ; \\
\Phi_{n+1} & =O\left(\epsilon^{2}+\epsilon^{2}\|z\|+\|z\|^{2}\right) .
\end{aligned}
$$

They derived stability conditions which ensure that, for arbitrary $\delta>0$, there exist $C_{1}, C_{2}$ and $\epsilon_{0}>0$ such that, for $0<\epsilon \leq \epsilon_{0}$, every solution of (1.11) satisfying $\left\|\mathbf{z}\left(t_{0}\right)\right\| \leq \delta \epsilon$, also satisfies $\|\mathbf{z}(t)\| \leq C_{2} \epsilon^{1 / 2}$, for $t_{0} \leq t \leq\left(t_{0}+C_{1} \epsilon^{-1 / 2}\right)$. The essence of the stability conditions is that they imply, for all eigenvalues $\lambda(\epsilon)$ of the matrix $A(\epsilon)$ given in (1.6), that $\operatorname{Re} \lambda(\epsilon) \leq-l \epsilon$, where $l>0$ is a constant.

Let

$$
B(\rho)=\left(\begin{array}{cccc}
a_{11}-\rho & \cdots & a_{1 n} & b_{1} \\
\cdots & \cdots & \cdots & \cdots \\
a_{n 1} & \cdots & a_{n n}-\rho & b_{n} \\
c_{1} & \cdots & c_{n} & 0
\end{array}\right) .
$$

Then, for $n>1$, the stability conditions given by Volosov and Morgunov [1] are that

$$
b_{i} c_{i}=-k^{2}<0,
$$

that all the roots of $\operatorname{det} B(\rho)=0$ should be distinct and have negative real parts, and that

$$
\sum_{i_{1}<i_{2}<\cdots<i_{n-s} \leq n} \Delta_{i_{1}, \cdots, i_{n-3}}-k^{2}\left(a_{i i}+h\right)>0 .
$$

Here $\Delta_{i_{1}}, \ldots, i_{n-\mathbf{s}}\left(i_{k}=1, \ldots, n\right)$ are the third order diagonal minors of $\operatorname{det} B(0)$, obtained by deleting the indicated rows and columns. For $n=1$ the stability conditions are just 


$$
b_{1} c_{1}<0 ; \quad\left(a_{11}+h\right)<0 \quad(n=1) .
$$

In this paper we confine our attention to the system (1.1), and give a detailed analysis of the motions in certain close neighborhoods of the stationary point $\alpha(\epsilon), \beta(\epsilon)$. In [2] we considered the case $n=1$, and investigated nonlinear oscillations in the neighborhood of the stationary point, with $(x-\alpha(\epsilon))=O\left(\epsilon^{1 / 2}\right)$ and $(y-\beta(\epsilon))=O(1)$. Here we consider $n>1$, and investigate slightly nonlinear oscillations in the neighborhood of the stationary point. We first suppose that $\|\mathbf{x}-\alpha(\epsilon)\|=O(\epsilon)$ and $(y-\beta(\epsilon))=O\left(\epsilon^{1 / 2}\right)$, and, after some preliminary transformations, apply the standard method of averaging [3]. It is found that the motion corresponding to the first order averaged equations is damped under the stability conditions given by Volosov and Morgunov, although we do not require that the roots of $\operatorname{det} B(\rho)=0$ be distinct. We obtain the condition (1.15) in the equivalent form

$$
\left(h-k^{-2} b_{i} c_{p} a_{p i}\right)<0 .
$$

The condition (1.14) implies that the motion is one of perturbed linear oscillations, the period of the oscillations being of order $\epsilon^{-1 / 2}$. We point out that our results show that, if the initial values are within order $\epsilon$ of the stationary point (and hence within order $\epsilon$ of the origin) then, although $(y-\beta(\epsilon))$ becomes of order $\epsilon^{1 / 2}$, in a time of order $\epsilon^{-1 / 2}$, $\|\mathbf{x}-\boldsymbol{\alpha}(\epsilon)\|$ remains of order $\epsilon$. Moreover, our averaged equations are valid for an interval of time $t$ of order $\epsilon^{-1}$, and $|y-\beta(\epsilon)| \leq O\left(\epsilon^{1 / 2}\right),\|\mathbf{x}-\alpha(\epsilon)\| \leq O(\epsilon)$ throughout that time.

We secondly assume that $(y-\beta(\epsilon))=O\left(\epsilon^{1 / 2}\right)$ and $\|\mathbf{x}-\alpha(\epsilon)\|=O\left(\epsilon^{1 / 2}\right)$, but subject to $c_{i}\left(x_{i}-\alpha_{i}(\epsilon)\right)=O(\epsilon)$. Note, from (1.8), that $\|\mathbf{x}\|=O\left(\epsilon^{1 / 2}\right)$ and $c_{i} x_{i}=O(\epsilon)$ imply that $Y^{(0)}(\mathbf{x})=O(\epsilon)$, and hence, from (1.1), that $d y / d t=O(\epsilon)$. The method of averaging is applied, after some preliminary transformation $\mathbf{S}$, and it is again found that the motion corresponding to the averaged equations is damped under the stability conditions given by Volosov and Morgunov, and the assumed order relations hold for a time interval $t$ of order $\epsilon^{-1}$. We emphasize, however, that we have investigated only the autonomous system (1.1) and, consequently, our results are less general, in this sense, than those of Volosov and Morgunov [1].

2. Motion in a certain close neighborhood. We first set

$$
x_{i}=\left[\alpha_{i}\left(\mu^{2}\right)+\mu^{2} \xi_{i}\right] ; \quad y=\left[\beta\left(\mu^{2}\right)+\mu \eta\right] ; \quad \tau=\mu t,
$$

where $\mu=\epsilon^{1 / 2}>0$, and $\alpha$ and $\beta$ have expansions as in (1.3). If $\operatorname{det} B(0) \neq 0$, where $B(\rho)$ is given by (1.13), then the equations

$$
X_{i}(\alpha(\epsilon), \beta(\epsilon) ; \epsilon)=0 ; \quad Y(\alpha(\epsilon), \beta(\epsilon) ; \epsilon)=0
$$

may be solved for $\alpha(\epsilon)$ and $\beta(\epsilon)$, for sufficiently small $\epsilon$. In particular, from (1.1)-(1.3), (1.7) and (1.8),

$$
a_{i j} \alpha_{j}^{(1)}+b_{i} \beta^{(1)}+X_{i}^{(2)}(0,0)=0 ; \quad c_{j} \alpha_{i}^{(1)}+Y^{(1)}(0,0)=0 .
$$

Substituting (2.1) into (1.1), it is found that

$$
d \xi_{i} / d \tau=b_{i} \eta+\mu Z_{i}(\xi, \eta ; \mu) ; \quad d \eta / d \tau=c_{i} \xi_{i}+\mu N(\xi, \eta ; \mu),
$$

where

$$
\begin{aligned}
Z_{i}(\xi, \eta ; \mu) & =Z_{i}^{(1)}(\xi, \eta)+\mu Z_{i}^{(2)}(\xi, \eta)+\cdots, \\
N(\xi, \eta ; \mu) & =N^{(1)}(\xi, \eta)+\mu N^{(2)}(\xi, \eta)+\cdots,
\end{aligned}
$$


and

$$
Z_{i}^{(l)}(\mathbf{0}, 0)=0 ; \quad N^{(l)}(0,0)=0 \quad(l=1,2, \cdots) .
$$

In particular,

$$
Z_{i}^{(1)}(\xi, \eta)=\left(a_{i j} \xi_{j}+g_{i} \eta^{2}\right) ; \quad N^{(1)}(\xi, \eta)=h \eta,
$$

where

$$
g_{i}=\frac{1}{2}\left(\partial^{2} X_{i}^{(1)} / \partial y^{2}\right)(0,0) .
$$

We suppose that (1.14) holds and make the transformations

$$
\xi_{i}=\left[b_{i}(C \sin k \tau+D \cos k \tau)+K_{i}\right] ; \quad \eta=k(C \cos k \tau-D \sin k \tau),
$$

where $c_{i} K_{i}=0$. Then $C, D$ and $K_{i}$ are constants if $\mu=0$ in (2.4). In order to avoid a technical difficulty later on, we remove the redundancy associated with the $K_{i}$. Since, from (1.14), not all the $c_{i}$ are zero, we may suppose, without loss of generality, that $c_{1} \neq 0$. Accordingly,

$$
K_{1}=-\sigma_{\beta} K_{\beta} ; \quad \sigma_{\beta}=c_{\beta} / c_{1},
$$

where we adopt the convention that repeated greek suffices are summed from 2 to $n$ (whereas repeated latin suffices are summed from 1 to $n$ ). It is found, from (2.4)-(2.6), (2.8), (2.10) and (2.11), that

$$
\begin{aligned}
d C / d \tau= & \mu\left[h \cos k \tau(C \cos k \tau-D \sin k \tau)-c_{p} g_{p} \sin k \tau(C \cos k \tau-D \sin k \tau)^{2}\right. \\
& \left.-k^{-2} b_{j} c_{p} a_{p i} \sin k \tau(C \sin k \tau+D \cos k \tau)-k^{-2} c_{p}\left(a_{p \beta}-\sigma_{\beta} a_{p 1}\right) K_{\beta} \sin k \tau\right] \\
& +O\left(\mu^{2}\right), \\
d D / d \tau= & -\mu\left[h \sin k \tau(C \cos k \tau-D \sin k \tau)+c_{p} g_{p} \cos k \tau(C \cos k \tau-D \sin k \tau)^{2}\right. \\
& \left.+k^{-2} b_{i} c_{p} a_{p i} \cos k \tau(C \sin k \tau+D \cos k \tau)+k^{-2} c_{p}\left(a_{p \beta}-\sigma_{\beta} a_{p 1}\right) K_{\beta} \cos k \tau\right] \\
& +O\left(\mu^{2}\right),
\end{aligned}
$$

and

$$
\begin{aligned}
& d K_{\alpha} / d \tau=\mu\left[b_{i}\left(a_{\alpha i}+k^{-2} b_{\alpha} c_{p} a_{p j}\right)(C \sin k \tau+D \cos k \tau)\right. \\
& \left.+\left(k^{2} g_{\alpha}+b_{\alpha} c_{p} g_{p}\right)(C \cos k \tau-D \sin k \tau)^{2}+\nu_{\alpha \beta} K_{\beta}\right]+O\left(\mu^{2}\right),
\end{aligned}
$$

for $\alpha=2, \cdots, n$, where

$$
\nu_{\alpha \beta}=\left[\left(a_{\alpha \beta}+k^{-2} b_{\alpha} c_{p} a_{p \beta}\right)-\sigma_{\beta}\left(a_{\alpha 1}+k^{-2} b_{\alpha} c_{p} a_{p 1}\right)\right] .
$$

Of course, if $n=1$ then $K_{1}=0$, and no $K_{\alpha}$ is present.

We may now apply the method of averaging [3] to Eqs. (2.12)-(2.14). That is, we assume an asymptotic expansion of the form

$$
\begin{aligned}
C & =\mathfrak{C}+\mu C^{(1)}(\mathfrak{C}, \mathfrak{D}, \mathfrak{K} ; \tau)+O\left(\mu^{2}\right), \\
D & =\mathfrak{D}+\mu D^{(1)}(\mathfrak{C}, \mathfrak{D}, \mathfrak{K} ; \tau)+O\left(\mu^{2}\right), \\
K_{\alpha} & =\Re_{\alpha}+\mu K_{\alpha}^{(1)}(\mathbb{C}, \mathfrak{D}, \mathfrak{K} ; \tau)+O\left(\mu^{2}\right),
\end{aligned}
$$

where $C^{(1)}, D^{(1)}$ and $K_{\alpha}^{(1)}$ are periodic in $\tau$, with period $2 \pi k^{-1}$, and 


$$
\begin{aligned}
d \mathfrak{C} / d \tau & =\mu P^{(1)}(\mathfrak{C}, \mathfrak{D}, \mathfrak{X})+O\left(\mu^{2}\right) ; \\
d \mathfrak{D} / d \tau & =\mu Q^{(1)}(\mathbb{C}, \mathfrak{D}, \mathfrak{C})+O\left(\mu^{2}\right) ; \\
d K_{\alpha} / d \tau & =\mu R_{\alpha}^{(1)}(\mathbb{C}, \mathfrak{D}, \mathfrak{X})+O\left(\mu^{2}\right) .
\end{aligned}
$$

It is a straightforward matter to substitute from (2.16) and (2.17) into (2.12)-(2.14), and equate the coefficients of $\mu$ on each side of the equations. In particular, $R_{\alpha}^{(1)}(\mathbb{C}, \mathfrak{D}, \mathfrak{K})+\left(\partial K_{\alpha}^{(1)} / \partial \tau\right)(\mathcal{C}, \mathfrak{D}, \mathfrak{K} ; \tau)=\left[b_{i}\left(a_{\alpha i}+k^{-2} b_{\alpha} c_{p} a_{p_{i}}\right)(\mathcal{C} \sin k \tau+\mathfrak{D} \cos k \tau)\right.$

$$
\left.+\left(k^{2} g_{\alpha}+b_{\alpha} c_{p} g_{p}\right)(\mathcal{C} \cos k \tau-\mathfrak{D} \sin k \tau)^{2}+\nu_{\alpha \beta} \Re_{\beta}\right] \text {. }
$$

Averaging (2.18) over a period of $\tau$, it follows that

$$
R_{\alpha}^{(1)}=\left[\nu_{\alpha \beta} \mathcal{K}_{\beta}+\frac{1}{2}\left(k^{2} g_{\alpha}+b_{\alpha} c_{p} g_{p}\right)\left(\mathfrak{C}^{2}+\mathfrak{D}^{2}\right)\right] .
$$

Now $K_{\alpha}^{(1)}$ may be determined (to within an arbitrary function of $\mathcal{C}$, $\mathfrak{D}$, and $\mathfrak{K}$ ) from (2.18), but we do not write out the expression here. Similarly, it is found that

$$
P^{(1)}=\frac{1}{2}\left(h-k^{-2} b_{j} c_{p} a_{p_{i}}\right) \mathcal{C} ; \quad Q^{(1)}=\frac{1}{2}\left(h-k^{-2} b_{j} c_{p} a_{p_{j}}\right) \text { D. }
$$

Let us consider the first order averaged equations, wherein the $O\left(\mu^{2}\right)$ terms in (2.17) are neglected. These equations will give $\mathfrak{C}, \mathbb{D}$ and $\mathfrak{x}$ to within $O(\mu)$, on an interval of $\tau$ of order $\mu^{-1}$ (so that the interval of time $t$ is of order $\mu^{-2}=\epsilon^{-1}$ ). We comment that, if the expansions in (2.16) are truncated so that the $O\left(\mu^{2}\right)$ terms are omitted, then the $O\left(\mu^{2}\right)$ terms in (2.17) will be dependent on $\tau$, but periodically so. The explicit dependence of the averaged equations on $\tau$ may be removed, of course, to any finite power of $\mu$, provided that the functions entering the original equations are sufficiently smooth. We now determine conditions under which the first order averaged equations correspond to damped motion. Thus, from (2.17) and (2.20), $\mathcal{C}$ and $\mathbb{D}$ will decay exponentially if

$$
\left(h-k^{-2} b_{i} c_{p} a_{p_{i}}\right)<0 .
$$

For $n=1$ this, in addition to (1.14), suffices and we are led to (1.16). For $n>1$, if (2.21) holds, then, from (2.17) and (2.19), $K_{\alpha}(\alpha=2, \cdots, n)$ will be damped if the real parts of all the roots of $\operatorname{det}[E(\rho)]=0$ are negative, where

$$
E_{\alpha \beta}=\left(\nu_{\alpha \beta}-\rho \delta_{\alpha \beta}\right) \text {, }
$$

and $\delta_{\alpha \beta}$ is a Kronecker delta. Note that we do not require that the roots be distinct. We will now establish the equivalence of (1.15) and (2.21), and also show that

$$
k^{2} \operatorname{det}[E(\rho)]=\operatorname{det}[B(\rho)],
$$

where $B(\rho)$ is given by (1.13). Thus, since $b_{i} c_{i}=-k^{2},(1.15)$ is equivalent to

$$
\begin{aligned}
0 & <\sum_{i, j=1}^{n} b_{j} c_{j} a_{i i}-k^{2} h+\sum_{i<j \leq n}\left[b_{i}\left(c_{j} a_{i i}-c_{i} a_{i j}\right)+b_{i}\left(c_{i} a_{i i}-c_{i} a_{i i}\right)\right] \\
& =\sum_{i, j=1}^{n} b_{j} c_{i} a_{i i}-k^{2} h+\sum_{i \neq j \leq n}\left(b_{i} c_{i} a_{i i}-b_{j} c_{i} a_{i i}\right) \\
& =\sum_{i, i=1}^{n} b_{j} c_{i} a_{i i}-k^{2} h .
\end{aligned}
$$

Next, from (1.13), 


$$
\operatorname{det}[B(\rho)]=\left|\begin{array}{ll}
L & b \\
c & 0
\end{array}\right|
$$

where $b$ is an $n$-column, $c$ is an $n$-row, and $L$ is an $n \times n$ matrix with

$$
L_{i j}=\left(a_{i j}-\rho \delta_{i j}\right) \text {. }
$$

By straightforward manipulations it follows that

$$
\left|\begin{array}{ll}
L & b \\
c & 0
\end{array}\right|=\left|\begin{array}{ll}
M & b \\
c & 0
\end{array}\right|=\left|\begin{array}{ll}
N & b \\
c & 0
\end{array}\right|
$$

where

$$
M_{i i}=\left[\left(a_{i i}+k^{-2} b_{i} c_{p} a_{p i}\right)-\rho \delta_{i i}\right]
$$

and

$$
N_{i j}=\left\{\left[\left(a_{i j}+k^{-2} b_{i} c_{p} a_{p i}\right)-\left(c_{j} / c_{1}\right)\left(a_{i 1}+k^{-2} b_{i} c_{p} a_{p 1}\right)\right]-\rho \delta_{i j}\right\} .
$$

Note, from (2.11), (2.15) and (2.22), that

$$
N_{\alpha 1}=0 ; \quad N_{\alpha \beta}=E_{\alpha \beta} \quad(\alpha, \beta=2, \cdots, n) .
$$

Also, it is readily found from (1.14) and (2.29) that

$$
c_{i} N_{i j}+\rho c_{i}=0 \quad(j=1, \cdots, n) .
$$

Hence (2.23) follows from (1.14), (2.25), (2.27), (2.30) and (2.31).

3. Motion in a wider neighborhood. We now return to the system (1.1) and set

$$
x_{i}=\left[\alpha_{i}\left(\mu^{2}\right)+\mu w_{i}\right] ; \quad y=\left[\beta\left(\mu^{2}\right)+\mu \eta\right] ; \quad \tau=\mu t,
$$

where

$$
c_{i} w_{i}=\mu \zeta,
$$

with $\zeta=O(1)$. Let

$$
r_{i j}=\frac{\partial^{2} X_{i}^{(1)}}{\partial x_{i} \partial y}(0,0) ; \quad s_{i i l}=\frac{1}{2} \frac{\partial^{2} X_{i}^{(1)}}{\partial x_{i} \partial x_{l}}(0,0)
$$

and

$$
p_{i j}=\frac{1}{2} \frac{\partial^{2} Y^{(0)}}{\partial x_{i} \partial x_{i}}(0) ; \quad q_{i j l}=\frac{1}{6} \frac{\partial^{3} Y^{(0)}}{\partial x_{i} \partial x_{i} \partial x_{l}}(0) .
$$

Then, substituting (3.1) into (1.1), and using (1.7)-(1.9), (2.3), (2.9), and (3.2)-(3.4), it is found that

$$
d w_{i} / d \tau=\mu\left(b_{i} \eta+a_{i j} w_{i}\right)+\mu^{2}\left(g_{i} \eta^{2}+r_{i j} w_{i} \eta+s_{i j l} w_{i} w_{l}\right)+O\left(\mu^{3}\right)
$$

and

$$
d \eta / d \tau=\left(\zeta+p_{i j} w_{i} w_{i}\right)+\mu\left(h \eta+d_{i} w_{i}+q_{i j l} w_{i} w_{i} w_{l}\right)+O\left(\mu^{2}\right) .
$$

If we eliminate $w_{1}$, using (3.2) and assuming that $c_{1} \neq 0$, then (3.5) and (3.6), using (1.14), lead to 


$$
d w_{\alpha} / d \tau=\mu\left[b_{\alpha} \eta+\left(a_{\alpha \beta}-\sigma_{\beta} a_{\alpha 1}\right) w_{\beta}\right]+O\left(\mu^{2}\right),
$$

$\left[\left(d \zeta / d \tau+k^{2} \eta\right)-c_{i}\left(a_{i \alpha}-\sigma_{\alpha} a_{i 1}\right) w_{\alpha}\right]$

$$
=\mu c_{i}\left[a_{i 1}\left(\zeta / c_{1}\right)+g_{i} \eta^{2}+\left(r_{i \alpha}-\sigma_{\alpha} r_{i 1}\right) w_{\alpha} \eta+u_{i \alpha \beta} w_{\alpha} w_{\beta}\right]+O\left(\mu^{2}\right),
$$

$\left[\left(d_{\eta} / d \tau-\zeta\right)-f_{\alpha \beta} w_{\alpha} w_{\beta}\right]$

$$
=\mu\left[h \eta+\left(d_{\alpha}-\sigma_{\alpha} d_{1}\right) w_{\alpha}+2\left(p_{1 \alpha}-\sigma_{\alpha} p_{11}\right) w_{\alpha}\left(\zeta / c_{1}\right)+v_{\alpha \beta \gamma} w_{\alpha} w_{\beta} w_{\gamma}\right]+O\left(\mu^{2}\right),
$$

where $\sigma_{\alpha}=\left(c_{\alpha} / c_{1}\right)$ and

$$
\begin{aligned}
f_{\alpha \beta} & =\left(p_{\alpha \beta}-2 \sigma_{\alpha} p_{1 \beta}+\sigma_{\alpha} \sigma_{\beta} p_{11}\right), \\
u_{i \alpha \beta} & =\left(s_{i \alpha \beta}-2 \sigma_{\alpha} s_{i 1 \beta}+\sigma_{\alpha} \sigma_{\beta} s_{i 11}\right), \\
v_{\alpha \beta \gamma} & =\left(q_{\alpha \beta \gamma}-3 \sigma_{\alpha} q_{1 \beta \gamma}+3 \sigma_{\alpha} \sigma_{\beta} q_{11 \gamma}-\sigma_{\alpha} \sigma_{\beta} \sigma_{\gamma} q_{111}\right),
\end{aligned}
$$

for $i=1, \cdots, n$, and $\alpha, \beta, \gamma=2, \cdots, n$.

We make the transformations

$$
\begin{aligned}
\zeta & =\left[k(F \cos k \tau-G \sin k \tau)-f_{\alpha \beta} w_{\alpha} w_{\beta}\right] ; \\
\eta & =\left[(F \sin k \tau+G \cos k \tau)+k^{-2} c_{i}\left(a_{i \alpha}-\sigma_{\alpha} a_{i 1}\right) w_{\alpha}\right] .
\end{aligned}
$$

Then (3.8) and (3.9), using (3.7), leads to

$$
\begin{aligned}
& d F / d \tau=\mu\left(\Psi \sin k \tau+k^{-1} \Phi \cos k \tau\right)+O\left(\mu^{2}\right) ; \\
& d G / d \tau=\mu\left(\Psi \cos k \tau-k^{-1} \Phi \sin k \tau\right)+O\left(\mu^{2}\right),
\end{aligned}
$$

where

$$
\begin{aligned}
\Phi=\left\{c_{i} a_{i 1}\left(\zeta / c_{1}\right)+c_{i} g_{i} \eta^{2}+\left[c_{i}\left(r_{i \alpha}-\sigma_{\alpha} r_{i 1}\right)+2 f_{\alpha \beta} b_{\beta}\right] w_{\alpha} \eta\right. \\
\left.+\left[c_{i} u_{i \alpha \beta}+f_{\beta \gamma}\left(a_{\gamma \alpha}-\sigma_{\alpha} a_{\gamma 1}\right)\right] w_{\alpha} w_{\beta}\right\},
\end{aligned}
$$

and

$$
\begin{aligned}
\Psi=\{ & {\left[h-k^{-2} b_{\alpha} c_{i}\left(a_{i \alpha}-\sigma_{\alpha} a_{i 1}\right)\right] \eta+2\left(p_{1 \alpha}-\sigma_{\alpha} p_{11}\right) w_{\alpha}\left(\zeta / c_{1}\right) } \\
& \left.+\left[\left(d_{\alpha}-\sigma_{\alpha} d_{1}\right)-k^{-2} c_{i}\left(a_{i \beta}-\sigma_{\beta} a_{i 1}\right)\left(a_{\beta \alpha}-\sigma_{\alpha} a_{\beta 1}\right)\right] w_{\alpha}+v_{\alpha \beta \gamma} w_{\alpha} w_{\beta} w_{\gamma}\right\} .
\end{aligned}
$$

If $\Phi$ and $\Psi$ in (3.14), and $\eta$ in (3.7), are expressed in terms of $F, G$ and $w_{\alpha}(\alpha=2, \cdots, n)$, using (3.13), then we may apply the method of averaging to the system (3.7) and (3.14).

Now, from (3.13), fixing $F, G$ and $w_{\alpha}$, and averaging over a period $2 \pi k^{-1}$ of $\tau$,

$$
\begin{aligned}
\langle\eta\rangle & =k^{-2} c_{i}\left(a_{i \alpha}-\sigma_{\alpha} a_{i 1}\right) w_{\alpha}, \\
\langle\zeta \sin k \tau\rangle & =-\frac{1}{2} k G ; \quad\langle\zeta \cos k \tau\rangle=\frac{1}{2} k F, \\
\langle\eta \sin k \tau\rangle & =\frac{1}{2} F ; \quad\langle\eta \cos k \tau\rangle=\frac{1}{2} G, \\
\left\langle\eta^{2} \sin k \tau\right\rangle & =k^{-2} c_{i}\left(a_{i \alpha}-\sigma_{\alpha} a_{i 1}\right) w_{\alpha} F,
\end{aligned}
$$

and

$$
\left\langle\eta^{2} \cos k \tau\right\rangle=k^{-2} c_{i}\left(a_{i \alpha}-\sigma_{\alpha} a_{i 1}\right) w_{\alpha} G .
$$

Hence, the first order averaged equations corresponding to (3.7) and (3.14) are, from (3.15)-(3.21), 


$$
d W_{\alpha} / d \tau=\mu \nu_{\alpha \beta} W_{\beta},
$$

where $\nu_{\alpha \beta}$ is given by (2.15), and

$$
d \mathfrak{F} / d \tau=\mu\left(\kappa \mathcal{F}+k^{-1} \rho_{\beta} W_{\beta} \mathcal{G}\right) ; \quad d \mathcal{G} / d \tau=\mu\left(\kappa \mathcal{G}-k^{-1} \rho_{\beta} W_{\beta} \mathcal{F}\right),
$$

where

$$
\kappa=\frac{1}{2}\left[h-k^{-2} b_{\alpha} c_{i}\left(a_{i \alpha}-\sigma_{\alpha} a_{i 1}\right)+c_{i} a_{i 1} / c_{1}\right],
$$

and

$$
\rho_{\beta}=\left[k^{-2} c_{i} c_{i} g_{j}\left(a_{i \beta}-\sigma_{\beta} a_{i 1}\right)+b_{\alpha} f_{\alpha \beta}+\frac{1}{2} c_{i}\left(r_{i \beta}-\sigma_{\beta} r_{i 1}\right)-k^{2}\left(p_{1 \beta}-\sigma_{\beta} p_{11}\right) / c_{1}\right] .
$$

From (1.14), (2.11) and (3.24) it follows that

$$
\kappa=\frac{1}{2}\left(h-k^{-2} b_{j} c_{i} a_{i j}\right) .
$$

Also, from (3.23),

$$
(d / d \tau)\left(\mathcal{F}^{2}+\mathcal{G}^{2}\right)=2 \mu \kappa\left(\mathcal{F}^{2}+\mathcal{G}^{2}\right) .
$$

Hence, from (3.22), (3.26) and (3.27), the motion corresponding to the first order averaged equations is damped under the same conditions as those obtained in the previous section, namely, if (2.21) holds and all the roots of $\operatorname{det}[E(\rho)]=0$ have negative real parts, where $E_{\alpha \beta}$ is given by (2.22). Let us examine the relationship between the analysis in the previous section and that in this one. From (2.1), (2.10) and (3.1),

$$
w_{i}=\mu\left[b_{i}(C \sin k \tau+D \cos k \tau)+K_{i}\right] .
$$

Also, from (1.14) and (3.2), since $c_{i} K_{i}=0$,

$$
\zeta=-k^{2}(C \sin k \tau+D \cos k \tau) .
$$

Now consider the case $w_{i}=O(\mu),(i=1, \cdots, n)$. Then, from (2.10), (3.13) and (3.29),

$$
F=-k D+O(\mu) ; \quad G=k C+O(\mu) .
$$

With $W_{\alpha}=O(\mu)$, it is seen from (2.20) and (3.26) that the first order averaged equations for $\mathcal{F}$ and $\mathcal{G}$ in (3.23) are consistent with the averaged equations for $\mathcal{C}$ and $D$ in (2.17). From (2.19), however, it is seen that the first order averaged equation for $W_{\alpha}$ in (3.22) is not consistent with the averaged equation for $\varkappa_{\alpha}$ in (2.17), but the reason for this is clear. Thus, the averaged equations for the slowly varying quantities $W_{\alpha}$ corresponding to $w_{\alpha}$ take the form

$$
d W_{\alpha} / d \tau=\mu \nu_{\alpha \beta}{ } W_{\beta}+O\left(\mu^{2}\right)
$$

and some of the $O\left(\mu^{2}\right)$ terms must be retained when $\mathfrak{W}_{\alpha}=\mu \mathcal{K}_{\alpha}$. Hence, in order to recover the result corresponding to (2.19), it is necessary to consider the second order averaged equations for $w_{\alpha}$.

\section{REFERENCES}

1. V. M. Volosov and B. I. Morgunov, Some stability conditions connected with resonance investigations, (in Russian), Dokl. Akad. NaukSSSR 170, 239-241 (1966). Soviet Math. Dokl. 7, 1159-1161 (1966)

2. J. A. Morrison, Slightly damped librations, Quart. Appl. Math. 24, 365-370 (1967)

3. N. N. Bogoliubov and Y. A. Mitropolsky, Asymptotic methods in the theory of nonlinear oscillations, Gordon and Breach, New York, 1961 